\title{
Radiation therapy and photodynamic therapy for biliary tract and ampullary carcinomas
}

\author{
Hiroya Saito ${ }^{1}$, Tadahiro Takada ${ }^{2}$, Masaru Miyazaki ${ }^{3}$, Shuichi Miyakawa ${ }^{4}$, Kazuhiro Tsukada ${ }^{5}$, \\ Masato Nagino ${ }^{6}$, Satoshi Kondo ${ }^{7}$, Juni Furuse $^{8}$, Toshio Tsuyuguchi ${ }^{9}$, Fumio Kimura ${ }^{3}$, Hideyuki Yoshitomi ${ }^{3}$, \\ Satoshi Nozawa ${ }^{3}$, Masahiro Yoshida ${ }^{2}$, Keita Wada $^{2}$, Hodaka Amano $^{2}$, and Fumihiko Miura ${ }^{2}$ \\ ${ }^{1}$ Department of Radiology, Asahikawa Kosei General Hospital, 1-24 Asahikawa 078-8211, Japan \\ ${ }^{2}$ Department of Surgery, Teikyo University School of Medicine, Tokyo, Japan \\ ${ }^{3}$ Department of General Surgery, Chiba University Graduate School of Medicine, Chiba, Japan \\ ${ }^{4}$ Department of Gastroenterological Surgery, Fujita Health University, Toyoake, Japan \\ ${ }^{5}$ Department of Surgery and Science, Graduate School of Medicine and Pharmaceutical Science for Research, University of Toyama, Toyama, \\ Japan \\ ${ }^{6}$ Division of Surgical Oncology, Department of Surgery, Nagoya University Graduate School of Medicine, Nagoya, Japan \\ ${ }^{7}$ Department of Surgical Oncology, Hokkaido University Graduate School of Medicine, Sapporo, Japan \\ ${ }^{8}$ Hepatobiliary and Pancreatic Oncology Division, National Cancer Center Hospital East, Chiba, Japan \\ ${ }^{9}$ Department of Medicine and Clinical Oncology, Chiba University Graduate School of Medicine, Chiba, Japan
}

\begin{abstract}
The purpose of radiation therapy for unresectable biliary tract cancer is to prolong survival or prolong stent patency, and to provide palliation of pain. For unresectable bile duct cancer, there are a number of studies showing that radiation therapy is superior to the best supportive care. Although radiation therapy is used in many institutions, no large randomized controlled trials (RCTs) have been performed to date and the evidence level supporting the superiority of this treatment is low. Because long-term relief of jaundice is difficult without using biliary stenting, a combination of radiation therapy and stent placement is commonly used. As radiation therapy, external-beam radiation therapy is usually performed, but combined use of intraluminal brachytherapy with external beam radiation therapy is more useful for making the treatment more effective. There are many reports demonstrating improved response rates as well as extended survival and time to recurrence achieved by this combination therapy. Despite the low level of the evidence, this combination therapy is performed at many institutions. It is expected that multiinstitutional RCTs will be carried out. Unresectable gallbladder cancer with a large focus is usually extensive, and normal organs with high radiosensitivity exist contiguously with it. Therefore, only limited anticancer effects are to be expected from external beam radiation therapy for this type of cancer. The number of reports on ampullary cancer is small and the role of radiation therapy in this cancer has not been established. Combination treatment for ampullary cancer consists of either a single use of intraoperative radiation therapy, postoperative external beam radiation therapy or intraluminal brachytherapy, or a combination of two or three of these therapies. Intraoperative radiation therapy is superior in that it enables precise irradiation to the target site, thereby protect-
\end{abstract}

Offprint requests to: $\mathrm{H}$. Saito

Received: October 1, 2007 / Accepted: October 22, 2007 ing adjacent highly radiosensitive normal tissues from irradiation. There are reports showing extended survival, although not significant, in groups undergoing intraoperative or postoperative radiation therapy compared with groups without radiation therapy. To date, there are no reports of large RCTs focusing on the significance of radiation therapy as a postoperative adjuvant treatment, so its usefulness as a postoperative adjuvant treatment is not proven. An alternative treatment is photodynamic therapy. There is an RCT demonstrating that, in unresectable bile duct cancer, extended survival and improved quality of life (QOL) have been achieved through a combination of photodynamic therapy and biliary stenting, compared with biliary stenting alone. Results from large RCTs are desired.

Key words External beam radiation therapy - Intraluminal brachytherapy $\cdot$ Intraoperative radiation therapy $\cdot$ Postoperative radiation therapy $\cdot$ Photodynamic therapy $\cdot$ Guidelines

\section{Introduction}

At present, the proportion of patients with curable early biliary tract cancer is small and the prognosis of this cancer remains poor as a whole. Early detection is a key element to improve its prognosis. However, the proportion of patients with advanced cancer is very large, so treatments are essential for residual lesions after resection and for nonresected patients. In biliary tract cancer, radiation therapy and other therapies are being performed for inoperative disease, for recurrence after resection, and for residual lesions after resection. Only few randomized controlled trials (RCTs) with large numbers of patients have been conducted, so standard radiation therapy for this cancer has not been established. 
Biliary tract cancers are low-radiosensitive adenocarcinomas and occur in deep-seated organs. Furthermore, normal tissues with higher radiosensitivity exist contiguously with the diseased sites. For these reasons, radiation therapy has not been considered as a good indication for these cancers. Since the prolonged survival achieved by 30 - to $60-$ Gy external beam radiation therapy was reported in the 1970s, in addition to intraoperative radiation therapy, postoperative radiation therapy, and intraluminal brachytherapy, combinations of external radiation therapy and chemotherapy have come into use. Subsequently, reports began to appear showing prolonged survival due to biliary tract drainage plus radiation therapy for unresectable cancers. Also, intraluminal radiation therapy combined with drainage tubes is being used actively to enhance anticancer effects. There are many reports demonstrating improved treatment results with this combination, and opinions supporting its usefulness are prevailing, despite the low level of evidence to prove its effectiveness.

A number of studies show the usefulness of radiation therapy for biliary tract cancer, but most of these studies are small prospective or retrospective studies, so studies that confer high-level evidence are limited. Thus, radiation therapy is not a standard treatment in biliary tract cancer. As a rule, pathological diagnosis should be determined prior to the institution of treatment. The frequency of occurrence of biliary tract cancer is low. Implementation of large multi-institutional RCTs is desired.

Here we pose clinical questions (CQs) on radiation therapy in biliary tract cancer, with responses in the form of recommendations (grades of the recommenda-

Table 1. Strength of recommendations ${ }^{1}$

A, Strongly recommend performing the clinical action

$\mathrm{B}$, Recommend performing the clinical action

$\mathrm{C} 1$, The clinical action may be considered although there is a lack of high-level scientific evidence for its use. May be useful

$\mathrm{C} 2$, Clinical action not definitively recommended because of insufficient scientific evidence. Evidence insufficient to support or deny usefulness

$\mathrm{D}$, Recommend not performing the clinical action tions are defined in Table $1^{1}$ ). Also, levels of evidence are given (in parentheses) for findings in reference citations (see definitions of levels in Table $2^{1}$ ).

CQ 1 Is radiation therapy recommended for patients with an unresectable biliary tract cancer?

For patients with an unresectable biliary tract cancer, radiation therapy may be used (recommendation C1).

The aim of radiation therapy for unresectable biliary tract cancer is to achieve prolonged survival or stent patency, and palliation of pain. Although there are many studies reporting the usefulness of, radiation therapy for this disease, the evidence level is low because no large RCTs have been conducted to date. ${ }^{2}$ The main symptom of biliary tract cancer is obstructive jaundice. However, radiation therapy is not short-acting, so priority is given to biliary drainage. On the other hand, long-term relief of jaundice is difficult without biliary stenting ${ }^{3}$ (level V) and a combination of stent placement and radiation therapy is used.

Only a small number of studies have reported radiation therapy for unresectable gallbladder cancer and ampullary cancer, so its significance is not sufficiently proven.

A large number of studies have reported a survival benefit with radiation therapy for unresectable bile duct cancer in comparison with other palliative treatments or best supportive care ${ }^{4,5}$ (level IV). External beam radiation therapy is a commonly used type of radiation therapy. External irradiation exceeding 50 Gy leads to the increased occurrence of complications in nearby organs such as digestive organs. In view of the higher frequency of low-radiosensitive adenocarcinomas in biliary tract cancer, a dosage of less than $50 \mathrm{~Gy}$ is not sufficient for local control. To achieve more effective radiation therapy, boosted treatment, such as intraluminal brachytherapy (see CQ 2) and intraoperative radiation therapy, should be administered. It is highly frequent that use of intraluminal brachytherapy through the drainage catheters in patients in whom an internal fistula has formed. ${ }^{6}$

Table 2. Levels of evidence ${ }^{1}$

\begin{tabular}{ll}
\hline Level I & Systematic review/meta-analysis \\
Level II & One or more randomized clinical trials \\
Level III & Nonrandomized controlled trials \\
Level IV & Analytic epidemiology (cohort studies and case-control studies) \\
Level V & Descriptive study (case reports and case-series studies) \\
Level VI & $\begin{array}{l}\text { Opinions of expert panels and individual experts not based on } \\
\text { patient's data }\end{array}$ \\
\hline
\end{tabular}


In unresectable bile duct cancer, many reports show the superiority of radiation therapy over the best supportive care. However, due to the lack of multiinstitutional RCTs, the strength of recommendation for radiation therapy is only $\mathrm{C} 1$ at present. Although standard radiation therapy has not been established in bile duct cancer, it is recommendable because prolonged survival is observed more often in patients with this cancer with a combination of external beam radiation therapy and intraluminal brachytherapy than with the single use of radiation therapy. ${ }^{6}$ The advantages of radiation therapy include prolonged stent patency and palliation of pain, with local cancer control. Explanations of the benefits and drawbacks of radiation therapy should be given to patients when decisions on treatment policies are made for those with incurable disease.

In unresectable biliary tract cancer, attempts at combining radiation therapy with chemotherapy are being made to enhance radiosensitivity in radiation therapy, to control metastatic lesions, and to prevent metastasis. However, there is no RCT comparing the single use of radiation therapy and its combined use with chemotherapy. Foo et al. $^{7}$ performed a small nonrandomized trial, in which the combination of external beam radiation therapy and intraluminal brachytherapy plus systemic administration of 5-fluorouracil (5-FU) was used; they found that an improved survival rate was achieved in the group for which the combination of chemotherapy and radiation therapy was used (level III). For combination regimens, 5-FU alone, 5-FU plus mitomycin $\mathrm{C}$ (MMC), MMC alone, and cisplatin (CDDP) plus 5-FU are reported (9-58 patients; median survival period, 830 months), ${ }^{2}$ but no recommendable regimen has been determined yet. Although there are many reports on the systemic administration of anticancer drugs for biliary tract cancer, intraarterial fusion chemotherapy has also been attempted.

There are reports demonstrating the efficacy of combinations of radiation therapy and chemotherapy for biliary tract cancer, but combining radiation therapy with chemotherapy as a standard is not always better than radiation therapy alone. In advanced cases of other gastrointestinal cancers, such as esophageal carcinoma ${ }^{8}$ and pancreatic cancer, ${ }^{9,10}$ there are studies showing that combination treatment of radiation therapy and chemotherapy is superior to radiation therapy alone, so the combination is thought to be a therapeutic modality worthy of consideration in biliary tract cancer. However, the efficacy of the combination of radiation therapy and chemotherapy for biliary tract cancer will have to be demonstrated by conducting RCTs at some future date.
CQ 2 Is intraluminal brachytherapy recommended for nonresected biliary tract cancer?

For radiation therapy of bile duct cancer, many reports demonstrate the usefulness of a combination of external beam radiation therapy with intraluminal brachytherapy than those seen with external beam radiation therapy alone (recommendation $\mathrm{C1}$ ).

External beam radiation therapy is generally used for bile duct cancer. Organs such as the digestive tract, liver, and kidney are included in the radiation field, so a dosage exceeding 50 Gy leads to the increased occurrence of complications. In general, a dosage exceeding 80 Gy enables tumor control, so boosted therapy such as intraluminal brachytherapy is required to deliver that dosage. ${ }^{6}$ Intraluminal brachytherapy has the advantage of enabling the delivery of a sufficient dosage to the target focus, while reducing the influence of irradiation on the surrounding tissues. Therefore, a combination of intraluminal brachytherapy and external beam radiation therapy contributes to increased effects of radiation therapy, with fewer adverse effects than those seen with external beam radiation therapy alone.

There are studies reporting that an improved response rate and an extended time to recurrence and survival were achieved by the combination of intraluminal brachytherapy and external beam radiation therapy for bile duct cancer ${ }^{6,11-15}$ (level IV). On the other hand, there are also studies that failed to find a difference in the survival period compared with that shown with external beam radiation therapy alone ${ }^{14-16}$ (level IV). Thus, there is a low level of evidence that the combination of external beam radiation therapy with intraluminal brachytherapy contributes to improved treatment results. However, opinions that the combination of intraluminal brachytherapy and external beam radiation therapy is an effective modality of radiation therapy for bile duct cancer are tending to prevail, and the combination is widely used in many institutions. In reality, most cases of bile duct cancer are advanced cancers and they are large in size. Therefore, improvement in the local control rate is not expected, but local control is possible with intraluminal brachytherapy in small-sized tumors ${ }^{17}$ (level V). Also, when the combination of external beam radiation therapy and intraluminal brachytherapy is used, attention should be paid to the occurrence of complications such as digestive tract hemorrhage and bile duct fistula. ${ }^{17,18}$

The combination of intraluminal brachytherapy and external beam radiation therapy (see Fig. 1 for representative results) for unresectable bile duct cancer confers a better survival rate than treatment with a single modality. ${ }^{2}$ However, due to the lack of large 

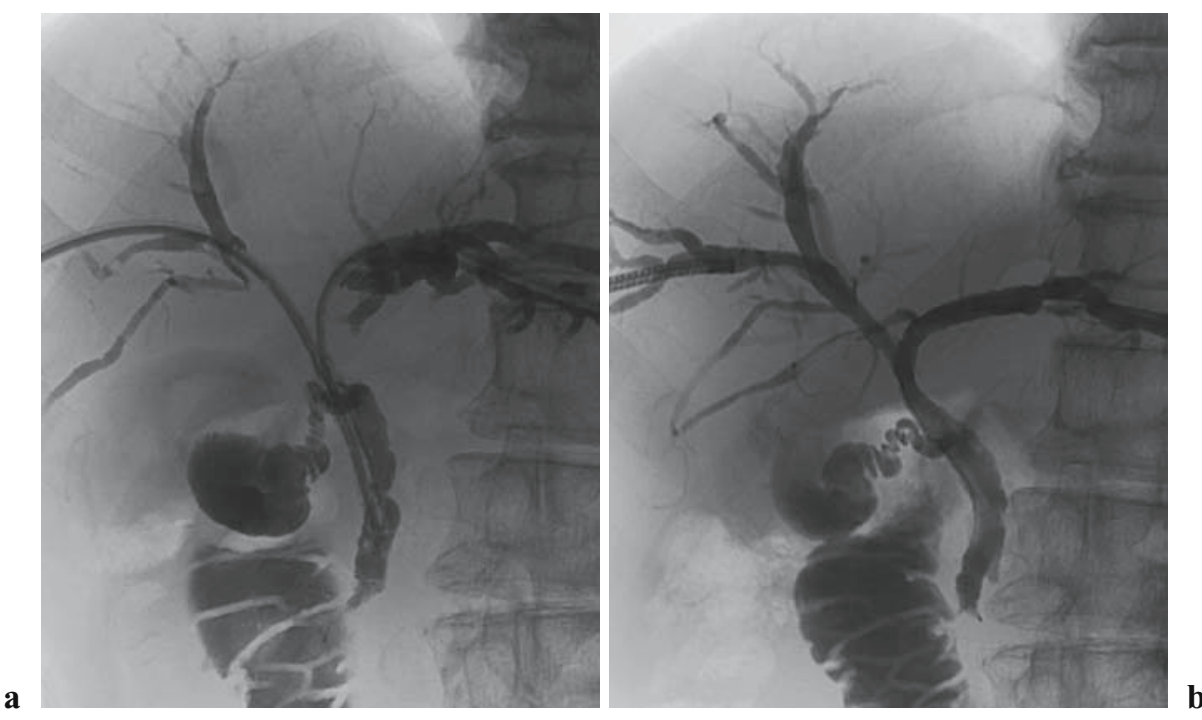

Fig. 1a,b. Percutaneous cholangiograms in a 67-year-old man with hilar bile duct carcinoma (Bismuth IV). a Before radiation therapy, cholangiogram shows complete obstruction of the bile duct at the bilateral hepatic duct and upper common bile duct. b After radiation therapy (external beam irradiation $44 \mathrm{~Gy}$ and intraluminal irradiation $15 \mathrm{~Gy}$ ), the bilateral hepatic duct and upper common bile duct wall is smooth, and passage is good
RCTs, the combination is not yet a standard therapy even though it is used in many institutions.

\section{CQ 3 Is intraoperative and/or postoperative radiation therapy recommended for resected biliary tract cancer?}

\section{Intraoperative and/or postoperative radiation therapy may be considered for patients with biliary tract cancer for whom noncurative resection has been conducted (recommendation C2).}

The rate of local recurrence is high even in patients with resected biliary tract cancer. Postoperative radiation therapy is occasionally performed for resected patients to improve the local control rate. However, the significance of postoperative adjuvant radiation therapy has not been reported in large controlled trials. In regard to the modality of radiation therapy employed, intraoperative radiation therapy, postoperative external beam radiation therapy, and/or intraluminal brachytherapy are performed, alone or in combination with each other. However, no consensus has been reached to date concerning which modality could be a standard adjuvant combination treatment after resection.

Pitt et al. ${ }^{19}$ conducted a prospective study of the efficacy of radiation therapy in 50 patients with bile duct cancer in whom they confirmed, during operation, that no distant metastasis was present. Comparison and discussion of treatment results were made by randomly assigning the 31 resected patients and 19 nonresected patients into a group of patients for whom radiation therapy was given $(n=23)$ and a group of patients for whom no radiation therapy was given $(n=27)$. A com- bination of external beam radiation therapy (mean total dosage, 46 Gy) and intraluminal brachytherapy with 192-Ir (mean dosage, 13 Gy) was administered. No significant extended survival or usefulness of the radiation therapy was observed, with the median survival period being 14 months for the treatment group and 15 months for the no-treatment group (level III). On the other hand, Gerhards et al. ${ }^{20}$ carried out operation alone for 20 patients, postoperative external beam radiation therapy alone for 30 patients (mean dosage, $46 \mathrm{~Gy}$ ), and a combination of postoperative external beam radiation therapy (mean dosage, $42 \mathrm{~Gy}$ ) and intraluminal brachytherapy (mean dosage, $10 \mathrm{~Gy}$ ) for 41 patients, in a total of 91 patients with resected bile duct cancer. They found significantly extended survival in the radiation group (24 months) compared with the nonradiation group (8 months) (level IV). Also, a report has demonstrated similar survival with postoperative radiation therapy for patients with cholangiocarcinoma with a positive biliary resection margin compared with those with a negative margin. $^{21}$

Intraoperative radiation therapy enables accurate irradiation to the target focus and exclusion of the surrounding high-radio-sensitive tissues from the irradiation field. Because increased dosage per irradiation and an enlarged irradiation field lead to the increased occurrence of complications, combined use of intraoperative radiation therapy with postoperative external beam radiation therapy is carried out. There are several studies reporting that prolonged survival was achieved by the combination treatment of intraoperative and postoperative irradiation in bile duct and gallbladder cancers ${ }^{22-25}$ (level IV).

In ampullary carcinoma, the role of radiation therapy is not known, because, as Abrams et al. ${ }^{26}$ reported in their study, a combination of radiation therapy with che- 
motherapy is often used, and patients with pancreatic cancers are included in most studies.

In the surgical treatment of biliary tract cancer, the resection that aims curability does not rare become incurable based on the final histopathological findings. ${ }^{26}$ However, no clinical studies have been conducted concerning postoperative radiation therapy for these patients, so no definite consensus has been reached. There are some studies demonstrating the usefulness of intraoperative and postoperative radiation therapy for resected cancers of the gallbladder and bile duct, but there is no evidence that can lead to the recommendation of radiation therapy as a standard treatment. Thus, radiation therapy may be considered for patients who have undergone noncurative resection.

It should be made clear by RCTs in future whether intraoperative and postoperative irradiation for patients who have undergone resection of biliary tract cancer contributes to an improved survival.

\section{CQ 4 Is photodynamic therapy recommended for} unresectable biliary tract cancer?

\section{There are reports demonstrating the usefulness of photodynamic therapy and it may be used for unresectable bile duct cancer (recommendation C1).}

For photodynamic therapy for unresectable bile duct cancer, there are two reports of RCTs and three reports of phase II studies.

The two reports of RCTs compared combination treatment of biliary tract stenting with photodynamic therapy and biliary tract stenting alone. Ortner et al. ${ }^{27}$ reported that significant prolongation was achieved in the median survival period (493 days vs 98 days) and that QOL was good (level II). However, the trial was discontinued because of the recommendation that biliary tract stenting alone failed to produce a good prognosis. Zoepf et al. ${ }^{28}$ reported significant prolongation of the median survival period (21 months vs 7 months) (level II). These two RCTs were small trials with 20 vs 19 patients and 16 vs 16 patients, respectively.

Three phase II studies of photodynamic therapy for unresectable bile duct cancer have been published. Berr et al. ${ }^{29}$ administered photodynamic therapy to 23 patients and found that a 6-month survival rate after diagnosis was achieved in $91 \%$ of the patients, a median survival period was 11 -month, the 30-day mortality rate was $4 \%$, and local tumor response (partial response [PR]) was achieved in $13 \%$; thus, they concluded that QOL was good and photodynamic therapy was useful (level IV). Dumoulin et al. ${ }^{30}$ treated 24 patients with photodynamic therapy and stenting and reported that the mean survival period was 15.9 months and median survival period was 9.9 months, but that no significant extended survival was achieved, compared with 12.5 months and 5.6 months, respectively, for the historical control group. However, according to their report, QOL was maintained in many patients, with 30-day and 60day mortality rates being $0 \%$ for both the patient and historical control groups (level IV). According to the authors' discussion, the absence of a significant difference in survival period may have been due to the markedly extended survival period achieved in the historical control group compared with that in the group receiving biliary tract stenting alone. In a study of 24 patients conducted by Shim et al., ${ }^{31}$ the 1-year survival rate was $59.6 \%$ and the median survival period was $558 \pm 178.8$ days. Furthermore, they found a significant reduction in the thickness of tumors measured by intraductal ultrasonography (IDUS), from $8.7 \pm 3.7 \mathrm{~mm}$ at 4 months before treatment to $5.8 \pm 2.0 \mathrm{~mm}$ at 4 months after treatment, and they also reported that improvement in the Karnofsky status was demonstrated (level IV).

In summary, combination treatment of photodynamic therapy and biliary tract stenting contributes to extended survival and improved QOL. However, it is not strongly recommended at this time due to the paucity of cases studied in RCTs. Results are awaited from large RCTs to be performed in the future.

Acknowledgment. We would like to express our deep gratitude to the members of the the Japanese Association of Biliary Surgery, the Japanese Society of HepatoBiliary-Pancreatic Surgery, and the Japan Society of Clinical Oncology, who provided us with great support and guidance in the preparation of the Guidelines. This process was conducted as part of the Integrated Research Project for Assessing Medical Technology 2005 and 2006 sponsored by the Japanese Ministry of Health, Labour, and Welfare.

We truly appreciate the following active working members who developed the draft of the evidencebased clinical practice Guidelines for the treatment of biliary tract cancer (Japanese version, 2007): Masahiro Kai (Miyazaki), Yasutoshi Kimura (Sapporo), Shigeaki Sawada (Toyama), Hiroaki Shimizu (Chiba), Hisatoshi Nakagawara (Kanazawa), Kohei Nakachi (Kashiwa), and Hiroyuki Yoshitome (Chiba). We also appreciate very much the following members who reviewed and approved the final Japanese version of the guidelines: Hiromitsu Saisyo (Ichikawa), Munemasa Ryu (Chiba), Satoru Shikata (Kyoto), and Yuji Nimura (Nagoya). 


\section{References}

1. Takada T, Miyazaki M, Miyakawa S, Tsukada K, Nagino M, Kondo S, et al. Purpose, use, and preparation of clinical practice guidelines for the management of biliary tract and ampullary carcinomas. J Hepatobiliary Pancreat Surg 2008;15:2-6.

2. Hejna M, Pruckmayer M, Raderer M. The role of chemotherapy and radiation in the management of biliary cancer: a review of the literature. Eur J Cancer 1998;34:977-86.

3. Ishii H, Furuse J, Nagase M, Kawashima M, Ikeda H, Yoshino M. Relief of jaundice by external beam radiation therapy and intraluminal brachytherapy in patients with extrahepatic cholangiocarcinoma: results without stenting. Hepatogastroenterology 2004;51: 954-7.

4. Grove MK, Hermann RE, Vogt DP, Broughan TA. Role of radiation after operative palliation in cancer of the proximal bile ducts. Am J Surg 1991;161:454-8.

5. Tollenaar RA, van de Velde CJ, Taat CW, Gonzalez Gonzalez D, Leer JW, Hermans J. External radiation therapy and extrahepatic bile duct cancer. Eur J Surg 1991;157:587-9.

6. Shin HS, Seong J, Kim WC, Lee HS, Moon SR, Lee IJ, et al. Combination of external beam irradiation and high-dose-rate intraluminal brachytherapy for inoperable carcinoma of the extrahepatic bile ducts. Int J Radiat Oncol Biol Phys 2003;57:105-12.

7. Foo ML, Gunderson LL, Bender CE, Buskirk SJ. External radiation therapy and transcatheter iridium in the treatment of extrahepatic bile duct carcinoma. Int J Radiat Oncol Biol Phys 1997:1;39:929-35.

8. Herskovic A, Martz K, al-Sarraf M, Leichman L, Brindle J, Vaitkevicius V, et al. Combined chemotherapy and radiation therapy compared with radiation therapy alone in patients with cancer of the esophagus. N Engl J Med 1992;326:1593-8.

9. Gastrointestinal Tumor Study Group. Treatment of locally unresectable carcinoma of the pancreas: comparison of combinedmodality therapy (chemotherapy plus radiation therapy) to chemotherapy alone. J Natl Cancer Inst 1988;80:750-5.

10. Okusaka T, Ito Y, Ueno H, Ikeda M, Takezako Y, Morizane C, et al. Phase II study of radiation therapy combined with gemcitabine for locally advanced pancreatic cancer. $\mathrm{Br} \mathrm{J}$ Cancer 2004;91:673-7.

11. Lu JJ, Bains YS, Abdel-Wahab M, Brandon AH, Wolfson AH, Raub WA, et al. High-dose-rate remote after loading intracavitary brachytherapy for the treatment of extrahepatic bile duct carcinoma. Cancer J 2002;8:74-8.

12. Fields JN, Emami B. Carcinoma of the extrahepatic biliary system: results of primary and adjuvant radiation therapy. Int J Radiat Oncol Biol Phys 1987;13:331-8.

13. Minsky BD, Kemeny N, Armstrong JG, Reichman B, Botet J. Extrahepatic biliary system cancer: an update of a combined modality approach. Am J Clin Oncol 1991;14:433-7.

14. Molt P, Hopfan S, Watson RC, Botet JF, Brennan MF. Intraluminal radiation therapy in the management of malignant biliary obstruction. Cancer 1986;57:536-44.

15. González González D, Gouma DJ, Rauws EA, van Gulik TM, Bosma A, Koedooder C. Role of radiation therapy, in particular intraluminal brachytherapy, in the treatment of proximal bile duct carcinoma. Ann Oncol 1999;10:215-20.

16. Morganti AG, Trodella L, Valentini V, Montemaggi P, Costamagna $\mathrm{G}$, Smaniotto D, et al. Combined modality treatment in unresectable extrahepatic biliary carcinoma. Int J Radiat Oncol Biol Phys 2000;46:913-9.
17. Takamura A, Saito H, Kamada T, Hiramatsu K, Takeuchi S, Hasegawa M, et al. Intraluminal low-dose-rate 192Ir brachytherapy combined with external beam radiation therapy and biliary stenting for unresectable extrahepatic bile duct carcinoma. Int J Radiat Oncol Biol Phys 2003;57:1357-65.

18. Kocak Z, Ozkan H, Adli M, Garipagaoglu M, Kurtman C, Cakmak A. Intraluminal brachytherapy with metallic stenting in the palliative treatment of malignant obstruction of the bile duct. Radiat Med 2005;23:200-207.

19. Pitt HA, Nakeeb A, Abrams RA, Coleman J, Piantadosi S, Yeo CJ, et al. Perihilar cholangiocarcinoma. Postoperative radiation therapy does not improve survival. Ann Surg 1995;221:788-98.

20. Gerhards MF, van Gulik TM, González González D, Rauws EA, Gouma DJ. Results of postoperative radiation therapy for resectable hilar cholangiocarcinoma. World J Surg 2003;27:173-9.

21. Stein DE, Heron DE, Rosato EL, Anné PR, Topham AK. Positive microscopic margins alter outcome in lymph node-negative cholangiocarcinoma when resection is combined with adjuvant radiation therapy. Am J Clin Oncol 2005;28:21-3.

22. Todoroki T, Iwasaki Y, Orii K, Otsuka M, Ohara K, Kawamoto T, et al. Resection combined with intraoperative radiation therapy (IORT) for stage IV (TNM) gallbladder carcinoma. World J Surg 1991;15:357-66.

23. Todoroki T, Iwasaki Y, Orii K, Otsuka M, Ohara K, Kawamoto T, et al. Benefits of combining radiation therapy with aggressive resection for stage IV gallbladder cancer. Hepatogastroenterology 1999;46:1585-91.

24. Gonzalez D, Gerard JP, Maners AW, De la Lande-Guyaux B, Van Dijk-Milatz A, Meerwaldt JH, et al. Results of radiation therapy in carcinoma of the proximal bile duct (Klatskin tumor). Semin Liver Dis 1990;10:131-41.

25. Langer JC, Langer B, Taylor BR, Zeldin R, Cummings B. Carcinoma of the extrahepatic bile ducts: results of an aggressive surgical approach. Surgery 1985;98:752-9.

26. Abrams RA, Grochow LB, Chakravarthy A, Sohn TA, Zahurak ML, Haulk TL, et al. Intensified adjuvant therapy for pancreatic and periampullary adenocarcinoma: survival results and observations regarding patterns of failure, radiation therapy dose and CA19-9 levels. Int J Radiat Oncol Biol Phys 1999;44:1039-46.

27. Ortner ME, Caca K, Berr F, Liebetruth J, Mansmann U, Huster $\mathrm{D}$, et al. Successful photodynamic therapy for nonresectable cholangiocarcinoma: a randomized prospective study. Gastroenterology 2003;125:1355-63.

28. Zoepf T, Jakobs R, Arnold JC, Apel D, Riemann JF. Palliation of nonresectable bile duct cancer: improved survival after photodynamic therapy. Am J Gastroenterol 2005;100:2426-30.

29. BerrF, Wiedmann M, Tannapfel A, Halm U, Kohlhaw KR, Schmidt F, et al. Photodynamic therapy for advanced bile duct cancer: evidence for improved palliation and extended survival. Hepatology 2000;31:291-8.

30. Dumoulin FL, Gerhardt T, Fuchs S, Scheurlen C, Neubrand M, Layer G, et al. Phase II study of photodynamic therapy and metal stent as palliative treatment for nonresectable hilar cholangiocarcinoma. Gastrointest Endosc 2003;57:860-7.

31. Shim CS, Cheon YK, Cha SW, Bhandari S, Moon JH, Cho YD, et al. Prospective study of the effectiveness of percutaneous transhepatic photodynamic therapy for advanced bile duct cancer and role of intraductal ultrasonography in response assessment. Endoscopy 2005;37:425-33. 